\title{
Non-Rigid 2D-3D Registration Using Anisotropic Error Ellipsoids to Account for Projection Uncertainties during Aortic Surgery
}

\author{
Alexis Guyot ${ }^{1, \star}$, Andreas Varnavas ${ }^{1}$, Tom Carrell $^{2}$, and Graeme Penney ${ }^{1}$ \\ 1 King's College London, Division of Imaging Sciences, London, UK \\ 2 Guy's \& St Thomas' NHS Foundation Trust, Dept. of Vascular Surgery, London, UK
}

\begin{abstract}
Overlay of preoperative images is increasingly being used to aid complex endovascular aortic repair and is obtained by rigid 2D-3D registration of $3 \mathrm{D}$ preoperative (CT) and 2D intraoperative (X-ray) data. However, for tortuous aortas large non-rigid deformations occur, thus a non-rigid registration must be performed to enable an accurate overlay. This article proposes the use of Thin-Plate Splines (TPS) to perform non-rigid 2D-3D registration. Intraoperative X-ray data contain no spatial information along the X-ray projection direction. Our approach accounts for this lack of spatial information by the use of an approximating TPS with non-isotropic error ellipsoids, where the major ellipsoid axis is aligned with the $\mathrm{X}$-ray projection direction. Experiments are carried out using 1D-2D and 2D-3D simulated data and 2D-3D interventional data. Simulated results show that our proposed method is 1.5 times more accurate than interpolating TPS based registration. Interventional data results show how large rigid registration errors of $9 \mathrm{~mm}$ can be reduced to $4 \mathrm{~mm}$ using our proposed method.
\end{abstract}

Keywords: Non-Rigid, 2D-3D registration, thin plate spline, non-isotropic errors.

\section{Introduction}

Complex endovascular aortic repair (EVAR), where the aneurysm extends over visceral vessels, is increasingly being used instead of open surgery. Complex EVAR is a technically challenging procedure requiring accurate $3 \mathrm{D}$ placement of often seven or more stent-grafts. The standard imaging protocol includes a contrast enhanced preoperative CT, showing $3 \mathrm{D}$ bony and vascular anatomy. The procedure is carried out using fluoroscopy guidance which provides 2D images of bony structures and interventional instruments, and 2D Digital Substraction Angiography (DSA) images that are used to visualise the blood vessels.

Due to the complex nature of these procedures, computer assistance has been proposed [1, 2], which involves rigid registration of the 3D preoperative data with the $2 \mathrm{D}$ intraoperative data, to enable $3 \mathrm{D}$ information from the preoperative

\footnotetext{
^ We are grateful to Philips Healthcare for funding this research.
} 
CT to be overlayed onto the intraoperative fluoroscopy (as seen in Fig. 5(a)]. However, in some cases (e.g. aortas with a neck angulation greater than $30^{\circ}$ 3]), inserting instruments leads to non-rigid deformation of the patient's aorta, which can result in large errors if a rigid registration method is used.

Several authors have proposed non-rigid 2D-3D registration to address this concern. Two such methods [4, 5] assume constant vessel length, and that deformations minimise a smoothness criterion. Liao et. al. 's method uses a 3D CT angiographic scan and proposes automatic segmentation of blood vessels from 2D DSA images, which is difficult to achieve robustly on clinical images. In addition, the deformations caused by stiff instruments inside the aorta are likely to violate the assumption of a minimum bending energy made by these two approaches.

We present a novel approach for 2D-3D non-rigid registration: Thin-Plate Splines (TPS) plus Projection Uncertainties (which we refer to as TPS+PU). After an initial rigid registration, aortic deformation is based on preoperatively determined fixed landmarks and a small number (less than 10) of manually identified moving landmarks in two fluoroscopy images. Unlike standard backprojection methods, our approach does not require corresponding landmarks to be identified in both fluoroscopy images. Such corresponding landmarks are difficult to find in clinical images. Instead our method models the lack of information perpendicular to the 2D fluoroscopy images using non-isotropic error ellipsoids, where the major axis lies along the back-projection lines.

\section{Theory}

Spline-based deformation algorithms have been widely used (see [6 6 ] for examples) for non-rigid registration when the source and target data have the same dimensionality, and are based upon the matching of source and target landmarks and minimisation of a smoothness criterion.

We propose a TPS-based registration method to register data sets with different dimensionality: a 3D preoperative CT to $2 \mathrm{D}$ intraoperative fluoroscopy. This configuration leads to projection uncertainties as the 3D position of landmarks selected from a 2D image cannot be determined within the 3D space. When two views are used, the $3 \mathrm{D}$ position of a landmark can be deduced from its corresponding $2 \mathrm{D}$ positions in both views. However, due to the nature of the images used during EVAR, corresponding landmarks are only rarely observed in both views, thus $3 \mathrm{D}$ reconstruction is generally not feasible.

Our method uses approximating TPS with non-isotropic errors [8] in a twoview scenario to cope with the aforementioned issue. As shown in Fig. 1, 3D source points $\left\{s_{i}\right\}$ and 2D target points $\left\{t_{i}^{2 D}\right\}$ are picked on the aorta surface and in the fluoroscopy images. 3D target points $\left\{t_{i}^{3 D}\right\}$ are calculated from $\left\{t_{i}^{2 D}\right\}$ as the closest point to $\left\{s_{i}\right\}$ on the back-projection line $\left\{l_{i}\right\}$ that joins $\left\{t_{i}^{2 D}\right\}$ to the X-ray source. Covariance matrices $\left\{\Sigma_{i}\right\}$ that represent the uncertainty in matching points $s_{i}$ to $t_{i}^{3 D}$ along lines $l_{i}$ are computed as follows: the major axis of the error ellipsoid is set to the direction of $l_{i}$ and to have length 1000 
(approximate fluoroscopy focal length) while the two other axes are set to zero length. Using these parameters for the ellipsoids and setting a weighting function $\lambda$ to 1 allows loose matching of landmarks along the back-projection lines. The following energy function, which weights the smoothness of the deformation $u=$ $\left(u_{1}, u_{2}, u_{3}\right)$ against its ability to match source and target points, is minimised:

$$
\begin{gathered}
J_{\lambda}(u)=\frac{1}{n} \sum_{i=1}^{n}\left(t_{i}^{3 D}-u\left(s_{i}\right)\right)^{T} \Sigma_{i}^{-1}\left(t_{i}^{3 D}-u\left(s_{i}\right)\right)+\lambda \sum_{k=1}^{3} J_{2}^{3}\left(u_{k}\right) \\
\text { where } J_{2}^{3}\left(u_{k}\right)=\sum_{\alpha_{1}+\alpha_{2}+\alpha_{3}=2} \frac{2}{\alpha_{1} ! \alpha_{2} ! \alpha_{3} !} \int_{R^{3}}\left(\frac{\partial^{2} u_{k}}{\partial x_{1}^{\alpha_{1}} \partial x_{2}^{\alpha_{2}} \partial x_{3}^{\alpha_{3}}}\right)^{2} d X
\end{gathered}
$$

and $n$ is the number of source landmarks. The analytical solution is:

$$
u_{k}(s)=\sum_{v=1}^{4} a_{k, v} \phi_{v}(s)+\sum_{i=1}^{n} w_{k, i} U\left(s, s_{i}\right) \text { for all } k \text { in }\{1,2,3\}
$$

with $s=\left(s_{x}, s_{y}, s_{z}\right)^{T}$ a point from the source image, $\phi_{1}(s)=1, \phi_{2}(s)=s_{x}$, $\phi_{3}(s)=s_{y}, \phi_{4}(s)=s_{z}$ and $U$ a suitable radial basis function. The $a_{k, v}$ and $w_{k, i}$ are sets of coefficients that respectively account for the rigid and the non-rigid part of $u_{k}$. Solution is obtained by solving the system:

$$
\begin{aligned}
\left(K+n \lambda W^{-1}\right) w+S a & =v \\
S^{T} w & =0
\end{aligned}
$$

where $K_{i j}=U\left(s_{i}, s_{j}\right), S_{i j}=\phi_{j}\left(s_{i}\right), W^{-1}=\operatorname{diag}\left(\Sigma_{1}, \ldots, \Sigma_{n}\right), v=\left(t_{1}^{T}, \ldots, t_{n}^{T}\right)$, with $t_{i}^{T}=\left(t_{i, x}^{3 D}, t_{i, y}^{3 D}, t_{i, z}^{3 D}\right), a=\left(a_{1}^{T}, \ldots, a_{4}^{T}\right)$, with $a_{v}^{T}=\left(a_{x, v}, a_{y, v}, a_{z, v}\right)$ and $w=\left(w_{1}^{T}, \ldots, w_{n}^{T}\right)$, with $w_{i}^{T}=\left(w_{x, i}, w_{y, i}, w_{z, i}\right)$.

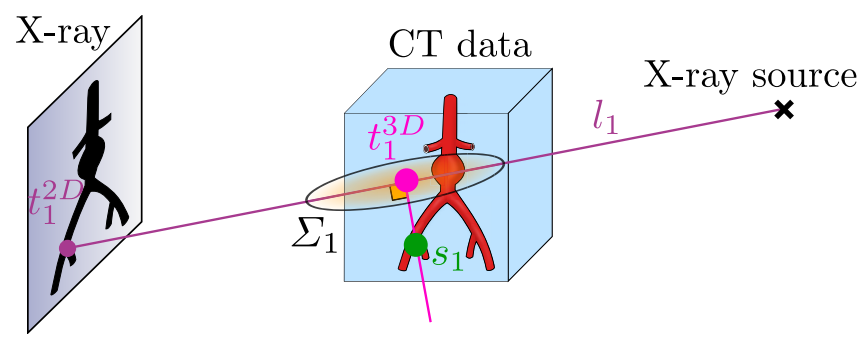

Fig. 1. Approximating TPS can be used to account for the uncertainty along the backprojection lines: 3D-3D TPS registration is performed using landmarks $\left\{s_{i}\right\}$ and $\left\{t_{i}^{3 D}\right\}$ with associated error ellipses with their major axes along the back-projection lines

The solution of this system is computed and applied to the aorta surface. We refer to this novel method as the 'single warp' strategy (see Fig. 2(left)). We compare it to a 'sequential warp' strategy which registers to each $2 \mathrm{D}$ image in turn and does not use non-isotropic error ellipsoids (see Fig. 2(right)). 

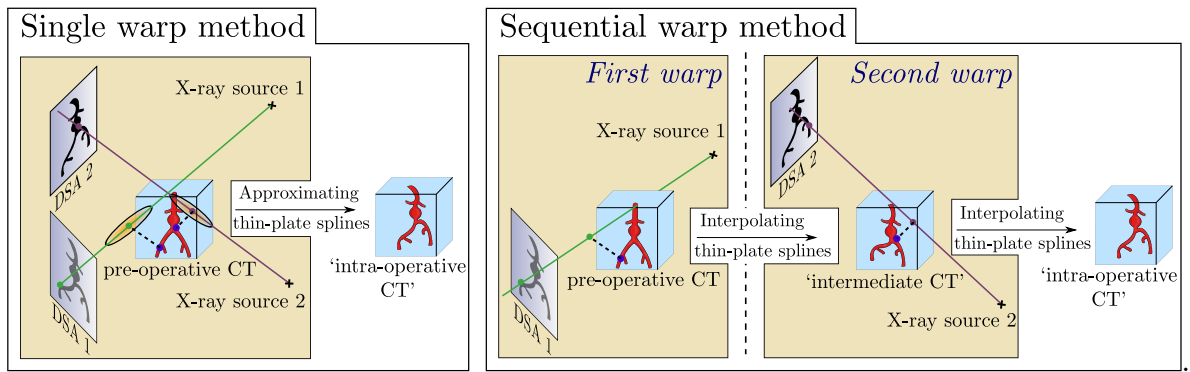

Fig. 2. Single warp strategy: registration is performed using target points from both views. Sequential warp strategy: a first registration is performed using target points from the first view, yielding an intermediate surface. A second registration is then performed using target points from the second view to warp the intermediate surface.

\section{$3 \quad$ Experiments}

\subsection{Data}

Three sets of data were used: (1) simulated 1D-2D synthetic data, (2) simulated 2D-3D data generated from interventional data, (3) 2D-3D interventional data.

Simulated 1D-2D synthetic data was produced to resemble an axial 2D slice of the aorta at the site of the renal arteries. This $2 \mathrm{D}$ slice is the simulated preoperative image (see Fig. 3 (a)), which was then warped to generate intraoperative datasets as follows: TPS transformations were carried out using five fixed points (the corners of a square around the aorta to anchor the deformation and the ostium of the left renal artery) and a single moving point at the right renal ostium which was displaced by $10 \mathrm{~mm}$ at an angle of $i \times 45^{\circ}, i=1, \ldots, 8$ for each $i^{\text {th }}$ deformation. From each of these warped images two 1D simulated intraoperative datasets were generated by a) projecting the ostia of the renal arteries onto one 1D plane (rr and lr in Fig. 3 (b)) and then b) projecting the edges of the aorta onto a second, $30^{\circ}$ rotated, 1D plane (ae and pe in Fig. $3(\mathrm{~b})$ ).

Simulated 2D-3D data generated from interventional data was produced using a patient CT as the preoperative data. Intraoperative data sets were generated by warping the CT image, eight times, using a TPS transformation. Fixed points were the corners of a large bounding box surrounding the abdominal aorta, the centres of vertebrae L1 to L5 and the bifurcations of the iliac arteries. Moving points were placed on the renal artery ostia and anterior and posterior edges of the aorta level with the renal arteries. These points were displaced 10mm. From each simulated 'intraoperative' CT scan, two intraoperative DSA images, with $30^{\circ}$ difference in view direction, were synthesised. 
2D-3D interventional data were used from a complex EVAR procedure. The data consisted of a preoperative CT scan, two intraoperative DSA images acquired after insertion of stent delivery device (views differed by $20^{\circ}$ rotation).

\subsection{Methods}

For each experiment, the aorta was deformed with either a single warp (TPS+PU, Fig 2(left)) or a sequential warp (interpolating TPS, Fig 2(right)). Each experiment used a set of fixed points (picked preoperatively) and moving points (picked from intraoperative images). The fixed points were as follows: for the 1D-2D synthetic experiment, corners of a square surrounding the aorta were fixed. For the 2D-3D experiments, corners of a large cube surrounding the aorta, centre of lumbar vertebrae and bifurcation of common iliac arteries were fixed.

Moving points for 1D-2D synthetic data were picked as illustrated in Fig. 3 (c). As in the case with real DSA images different information is visible in different views. We assume that the renal ostia are visible, and so can be picked, in plane 1 (equivalent to an anterior-posterior DSA), whereas only the outline of the aorta is likely to be visible from the rotated view, plane 2 .

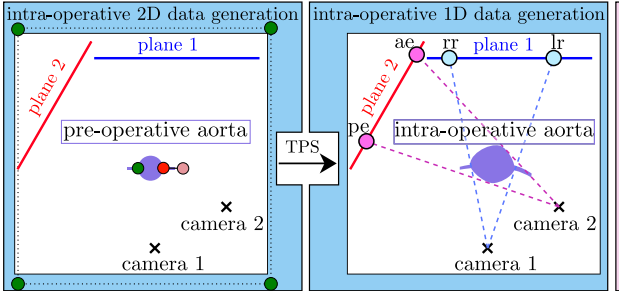

(a)

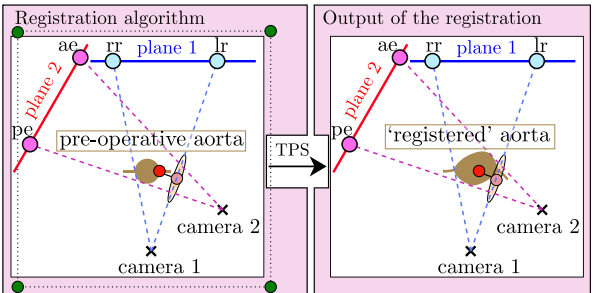

(c) (d)

(O) $1 \mathrm{D}$ target points $\circ 2 \mathrm{D}$ fixed points $\circ 2 \mathrm{D}$ source points $\circ 2 \mathrm{D}$ target points OError ellipses

Fig. 3. (a) interpolating TPS is applied to the preoperative aorta to obtain the intraoperative aorta (b) 1D intraoperative points: left, right renals (lr, rr) and anterior, posterior edges (ae, pe), are generated by projecting the intraoperative aorta onto the $1 \mathrm{D}$ planes. (c) 1D target points are selected on the 1D intraoperative planes and the points on the relevant aortic area closest to the back-projection lines are selected as corresponding $2 \mathrm{D}$ source points. The points on the back-projection lines closest to the $2 \mathrm{D}$ source points are selected as corresponding $2 \mathrm{D}$ target points. For the single warp, error ellipses are produced along the back-projection lines. (d) Result of applying non-rigid TPS + PU registration on the aorta using $2 \mathrm{D}$ source and $2 \mathrm{D}$ target points.

1D target points are selected on the 1D intraoperative planes and the corresponding points on the aorta are selected as $2 \mathrm{D}$ source points. For the renal positions corresponding points were the 2D renal artery ostia (see Fig. 3 (c)). For aortic edge positions corresponding points were the closest $2 \mathrm{D}$ edge points to back projection lines from the $1 \mathrm{D}$ point positions. 
Moving points for the simulated 2D-3D data were picked at the ostia of the renal arteries and the Superior Mesenteric Artery (SMA) on the first DSA image and at the outline of the aorta on the second DSA image. The location of corresponding 3D source and 3D target points was found in a process equivalent to the one presented in Fig. 3 (c).

Moving points for the interventional data were picked at the ostia of the vessels when visible, generating $3 \mathrm{D}$ source and $3 \mathrm{D}$ target points as in the case of simulated 2D-3D data. The rigidly-registered aortic CT surface was then overlayed onto the first DSA image and the location of largest displacement with respect to the aortic outline was located. An additional moving point was picked at this location, non-rigid registration was performed using all previously picked points and the displayed aortic overlay was updated. This process was repeated, generating one additional moving point at each iteration until the aortic overlay closely matched the DSA image (i.e. within $\approx 2 \mathrm{~mm}$ ). Moving points were selected in the second DSA image using the same procedure. Overall, 7 moving points were picked within approximately 2 minutes.

\subsection{Validation}

For simulated data experiments Target Registration Errors (TRE) were calculated to the known ground truth using the following regions of interest: ROI 1 was $26 \times 26 \mathrm{~mm}^{2}$ or $30 \times 30 \times 30 \mathrm{~mm}^{3}$ region centred on the aorta at the level of the renal arteries for the $1 \mathrm{D}-2 \mathrm{D}$ and $2 \mathrm{D}-3 \mathrm{D}$ data respectively. $R O I 2$ was a $2 \times 2 \mathrm{~mm}^{2}$ or $2 \times 2 \times 2 \mathrm{~mm}^{3}$ region centred on the right renal artery ostium (for the $2 \mathrm{D}-3 \mathrm{D}$ data $R O I 3$ denotes the left renal ostium).

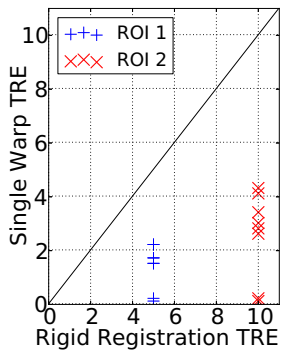

(a)

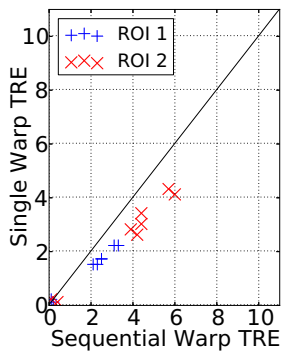

(b)

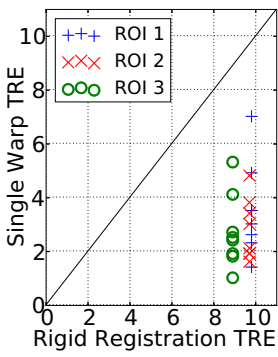

(c)

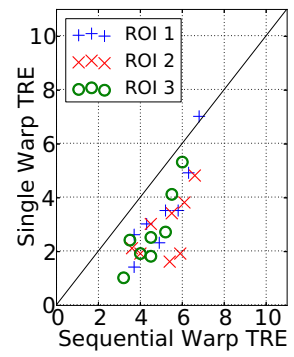

(d)

Fig. 4. All TRE values are expressed in mm. (a) 1D-2D synthetic data: Single Warp vs. Rigid Registration TRE (b) 1D-2D synthetic data: Single Warp vs. Sequential Warp TRE (c) 2D-3D synthetic data: Single Warp vs. Rigid Registration TRE (d) 2D-3D synthetic data: Single Warp vs. Sequential Warp TRE. 
No ground truth was available for the interventional data. Instead the position of the CT overlay was compared to a DSA image acquired later in the intervention. Reprojection error distances were calculated (as described in [2]) at the left ( $R O I 1)$ and right (ROI 2) renal artery ostia.

\section{Results}

Results comparing TRE values for rigid registration, single warp and sequential warp for the 1D-2D and 2D-3D synthesised data are shown in Fig. 4. The single warp results show a major improvement compared to rigid registration: registration accuracy is on average 3.8 times higher for the 1D-2D data and 3.1 times higher for the 2D-3D data. The single warp results also show an improvement compared to the sequential warp: registration accuracy is on average 1.4 times higher for the 1D-2D data and 1.6 times higher for the 2D-3D data.

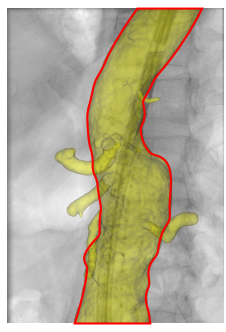

X-ray image (before warp)

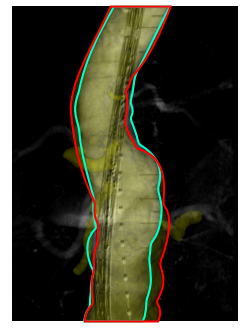

DSA image (before warp)

(a)

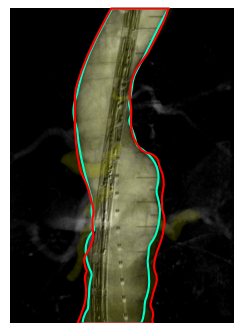

DSA image (after warp)

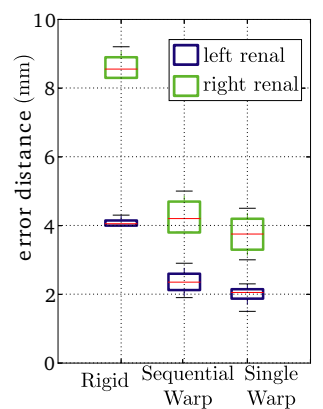

(b)

Fig. 5. (a) Comparison of CT overlay with DSA image before and after warp. Aorta outline from overlay (red) matches more accurately to DSA (cyan) after warp. (b) Registration accuracy at renal ostia using rigid registration, sequential and single warp.

Fig 5(a) visually shows the improvement in using the single warp compared to rigid registration on clinical data. Numerical results are presented in Fig. $5(\mathrm{~b})$ where applying the single warp halved the rigid registration error.

\section{Discussion and Conclusions}

Two approaches (sequential warp with interpolating TPS and single warp with $\mathrm{TPS}+\mathrm{PU})$ to deform the aorta were described and compared. Results on simulated data show a clear improvement in accuracy when using TPS+PU. A recent study [3] reported a range of up to $11 \mathrm{~mm}$ (mean $4.5 \mathrm{~mm}$ ) for aortic movement. Despite applying deformations of $10 \mathrm{~mm}$, almost at the top of this reported range, we managed to achieve the required registration accuracy less than $3 \mathrm{~mm}$ 
(as proposed in [3]) for half the single warp registrations. No sequential warp registration achieved $<3 \mathrm{~mm}$ accuracy (see Fig. 4(d)].

Our method fits well within the standard clinical workflow. It requires knowledgeable manual input for point picking, but only a few points are required, which are usually located in standard clinical images within a few minutes (compared to the procedure time of $4+$ hours). Automated landmark identification would be preferred, however this is very difficult to achieve with guaranteed $100 \%$ robustness, and subsequent required checks on automated landmark selection are likely to require knowledgeable manual visual inspection. 2D-3D rigid registration is performed regularly to account for rigid patient, and fluoroscopy gantry, movement during complex EVAR. Our current approach assumes that only one major non-rigid deformation (which occurs after inserting a stiff wire) takes place during the procedure. Therefore, the non-rigid registration algorithm would only need to be applied once per procedure. This assumption remains to be clinically verified.

In conclusion, we present a new non-rigid 2D-3D registration method using a TPS with non-isotropic error ellipsoids to model projection uncertainties. Results show improved accuracy using TPS+PU, which has the potential to increase the number of EVAR patients who can benefit from computer-assisted surgery.

\section{References}

1. Kaladji, A., Dumenil, A., Castro, M., Haigron, P., Heautot, J.F., Haulon, S.: Endovascular aortic repair of a postdissecting thoracoabdominal aneurysm using intraoperative fusion imaging. J. Vasc. Surg. 57, 1109-1112 (2013)

2. Penney, G., Varnavas, A., Dastur, N., Carrell, T.: An image-guided surgery system to aid endovascular treatment of complex aortic aneurysms: Description and initial clinical experience. In: Taylor, R.H., Yang, G.-Z. (eds.) IPCAI 2011. LNCS, vol. 6689, pp. 13-24. Springer, Heidelberg (2011)

3. Carrell, T., Modarai, B., Brown, J., Penney, G.: Feasibility and limitations of an automated 2D-3D rigid image registration system for complex endovascular aortic procedures. J. Endovasc. Ther. 17(4), 527-533 (2010)

4. Groher, M., Baust, M., Zikic, D., Navab, N.: Monocular deformable model-to-image registration of vascular structures. In: Fischer, B., Dawant, B.M., Lorenz, C. (eds.) WBIR 2010. LNCS, vol. 6204, pp. 37-47. Springer, Heidelberg (2010)

5. Liao, R., Tan, Y., Sundar, H., Pfister, M., Kamen, A.: An efficient graph-based deformable $2 \mathrm{D} / 3 \mathrm{D}$ registration algorithm with applications for abdominal aortic aneurysm interventions. In: Liao, H., Edwards, P.J., Pan, X., Fan, Y., Yang, G.-Z. (eds.) MIAR 2010. LNCS, vol. 6326, pp. 561-570. Springer, Heidelberg (2010)

6. Bazen, A.M., Gerez, S.H.: Fingerprint matching by thin-plate spline modelling of elastic deformations. Pattern Recogn. 36(8), 1859-1867 (2003)

7. Johnson, H.J., Christensen, G.E.: Landmark and intensity-based, consistent thinplate spline image registration. In: Insana, M.F., Leahy, R.M. (eds.) IPMI 2001. LNCS, vol. 2082, pp. 329-343. Springer, Heidelberg (2001)

8. Rohr, K., Stiehl, H., Sprengel, R., Buzug, T., Weese, J., Kuhn, M.: Landmark-based elastic registration using approximating thin-plate splines. IEEE Transactions on Medical Imaging 20(6), 526-534 (2001) 